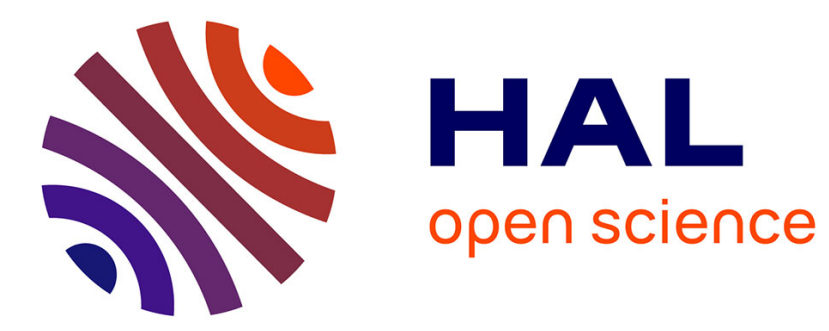

\title{
Xe NMR-based sensors: biological applications and recent methods
}

\author{
E Mari, P Berthault
}

\section{To cite this version:}

E Mari, P Berthault. Xe NMR-based sensors: biological applications and recent methods. Analyst, 2017, 142, pp.3298-3308. 10.1039/C7AN01088E . cea-01573931

\section{HAL Id: cea-01573931 https://hal-cea.archives-ouvertes.fr/cea-01573931}

Submitted on 11 Aug 2017

HAL is a multi-disciplinary open access archive for the deposit and dissemination of scientific research documents, whether they are published or not. The documents may come from teaching and research institutions in France or abroad, or from public or private research centers.
L'archive ouverte pluridisciplinaire HAL, est destinée au dépôt et à la diffusion de documents scientifiques de niveau recherche, publiés ou non, émanant des établissements d'enseignement et de recherche français ou étrangers, des laboratoires publics ou privés. 


\section{${ }^{129}$ Xe NMR-based sensors: biological applications and recent methods}

E. Mari ${ }^{\mathrm{a}}$ and P. Berthault ${ }^{\mathrm{a}}$

Xenon is a first-rate sensor of biological events, due to its large polarizable electron cloud inducing significant modification of NMR parameters through slight changes in its local environment. The use of xenon as a sensor is of increasing interest for sensitive magnetic resonance imaging, since its signal can be enhanced by several orders of magnitude, mainly by spinexchange optical pumping. Furthermore xenon can be vectorized toward targets of interest by using functionalized host systems, enabling their detection at subnanomolar concentrations. Associated with a new generation of detection methods this gives rise to a powerful molecular imaging approach, where xenon can be delivered on purpose several times after introduction of the functionalized host system. 


\section{Introduction}

From simple photograph of the inside of the human body providing information on bone structure or form and abnormalities of various organs, molecular imaging now offers a dynamic view of biological processes occurring locally, witnessing for instance the presence of infectious cells or metabolic deregulations. The development of powerful imaging techniques is the key to early diagnosis of disease, best follow-up treatments and also biomedical research tools. These techniques developed particularly in the 21th century form part of the molecular imaging concept.

Magnetic resonance imaging (MRI) is a good compromise to achieve molecular imaging in vivo in real time without any perturbative radiation. The major advantages of MRI are its potential high spatial resolution $(25-100 \mu \mathrm{m})$, its low invasiveness, its harmlessness and the excellent tissue contrast it can provide. In this context, MRI complements and sometimes overruns other molecular imaging approaches by enabling monitoring of events at the cellular or even subcellular level. However, classical implementation of this technique suffers from low sensitivity due to the very low population differences between nuclear spin energy levels at Boltzmann equilibrium. Hyperpolarization techniques ${ }^{1}$ that transiently increase the nuclear spin polarization of a very dilute agent by several orders of magnitude circumvent the MRI sensitivity problem. Among the species that can be spinhyperpolarized, xenon is of high interest, due to its exogenous nature (leading to the absence of background signal) and the fact that it can act as a spy of biological events without interfering with them. Moreover, it can be endlessly reloaded and simply removed from the sample since it is a gas at ambient temperature and pressure. Finally, owing to the high deformability of its large electron cloud xenon is deeply sensitive to its local environment and constitutes a perfect probe for various biological interactions. Soluble in most biological fluids, xenon can cross the plasma membrane in a few tens of milliseconds without losing its hyperpolarization. ${ }^{2}$ The initial applications of hyperpolarized xenon in biology were the anatomical imaging of the lung $^{3}$ and the investigation of hydrophobic binding pockets in proteins ${ }^{4,5}$ since the probe is mainly lipophilic. In this last domain, xenon provides information both through direct observation of its NMR spectrum and via transfer of its enhanced polarization to surrounding spins. A third possibility is the observation of chemical shift changes of the protein nuclei $\left({ }^{1} \mathrm{H},{ }^{15} \mathrm{~N}\right.$, or $\left.{ }^{13} \mathrm{C}\right)$ induced by xenon atoms in the hydrophobic cavity, leading to chemical shift mapping useful to extract the thermodynamics parameters of the interaction.

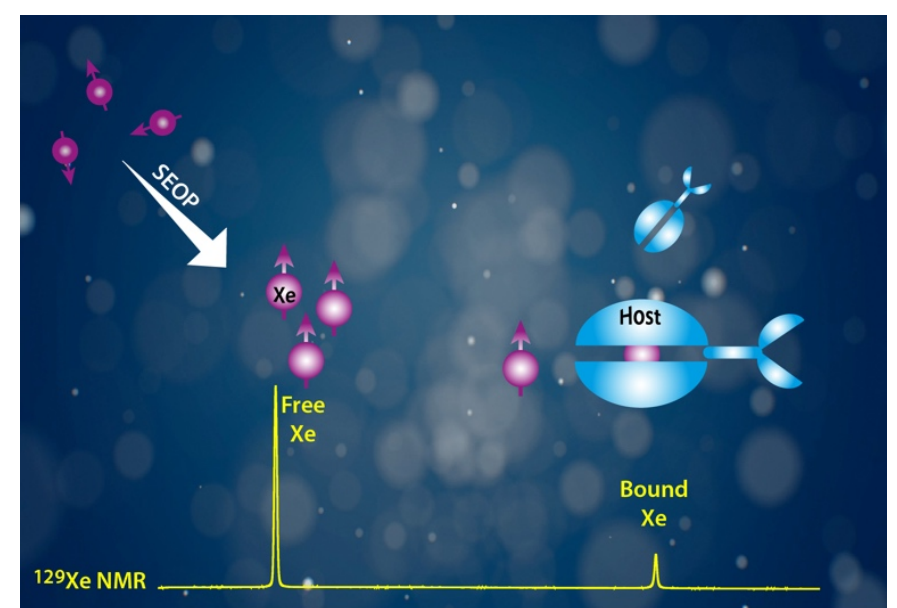

Fig. 1. Principle of the ${ }^{129}$ Xe NMR-based biosensing approach. Xenon is polarized by Spin-Exchange Optical Pumping (SEOP) and introduced into the sample containing xenon hosts. Its spectrum displays distinct signals for the free and bound forms.

In medical applications xenon is, at this stage, employed as anaesthetic for high-risk patients since 2007 in Europe and perceived as "the anaesthetic gas of the future". ${ }^{6}$ Even though the mechanism is unclear, the inhibition of N-methyl-Daspartate (NMDA) receptors by xenon has been viewed as a primary cause of xenon anaesthesia. Except this, xenon has no known affinity for any given receptor which means that it will disperse over the entire body, which is far from ideal for obtaining a contrast and studying a particular pathology or biological event of interest. To avoid this, hyperpolarized xenon can be targeted toward specific biological receptors or analytes through the design of functionalized molecular systems (Fig.1). These systems are composed of two parts: a molecular system or assembly reversibly trapping the noble gas and a tethered ligand designed to recognize a given biological function or chemical group.

This minireview presents the recent applications of these ${ }^{129} \mathrm{Xe}$ NMR-based sensors after a description of the use of hyperpolarized xenon, the different hyperpolarization techniques and the prerequisites of the functionalized systems. Finally, optimized schemes for detection of very small amount of xenon biosensors are discussed.

\section{Hyperpolarized xenon}

Hyperpolarization consists in transiently imbalancing the nuclear spin repartition of the energy level populations initially at the Boltzmann distribution, by polarization transfer from a more ordered system. In the case of xenon, it can be done via Optical Pumping (OP) or Dynamic Nuclear Polarization (DNP). 
A photon beam can be fully circularly polarized (spin +1 or -1 ), and therefore can be the ideal polarization source. However, as optical transitions do not influence the nuclear spins directly (only the rearrangement of the orbital motion of an electron can be responsible for photon absorption), the experiment is a two-step process. The Spin-Exchange Optical Pumping (SEOP) experiment involves an alkali metal that is heated so that the gas phase absorbs circularly polarized photons at the wavelength corresponding to the transition between its ground state and its first excited state (795 nm for rubidium). The optical pumping cell is placed in a magnetic field (defining the quantization axis) colinear to the light beam in order to split the degeneracy of the electron spin levels. This first step is extremely fast and complete hyperpolarization of the electron spins occurs in microsecond to millisecond time scale depending on the experimental conditions. As the cell also contains the noble gas, a second step occurs, consisting in polarization transfer from the electron spins of the alkali metal to the xenon nuclear spins. This second step, based on crossrelaxation phenomenon during the transient formation of van der Waals Rb-Xe pairs or during xenon-alkali metal collisions, is obviously less efficient and slower than the first one. In our experimental conditions (non-narrowed laser diodes, pressure inside the pumping cell beyond 1 bar) the typical time scale for obtaining a fully hyperpolarized nuclear spin system is on the order of one to five minutes for a $12-\mathrm{mL}$ batch.

The procedure for hyperpolarizing xenon via DNP includes a first production of an amorphous solid pellet containing xenon in an adequate frozen solvent doped with free radicals. In this solid state, the high electron spin polarization due to the combined use of low temperature and high static magnetic field is transferred in part to the nuclear spins by microwave irradiation in some tens of minutes. Then a fast sublimation step ensures separation of the noble gas from the radicals.

Thus, time to produce hyperpolarized xenon via SEOP advantageously compares with the characteristic time scale of DNP, which, according to Capozzi et al. is about $1 \mathrm{hr}$ and half for a $80 \mathrm{~mL}$ batch. $^{7}$

While for DNP it is easier to increase the hyperpolarized xenon quantities by increasing the solid-state sample volume, the SEOP experiment is cheaper and prone to provide in a short time xenon with a polarization exceeding 0.2 , in quantities enabling in vitro and in vivo (mainly for small animals) NMR/MRI studies.

For biomedical applications using laser-polarized xenon, a concern has to be addressed dealing with the chemical species other than xenon inside the optical pumping cell. In addition to the noble gas, the cell contains some droplets of the alkali metal, a few hundred torrs of nitrogen (a quenching gas designed to avoid radiative de-excitation susceptible to give rise to photons with opposite polarization) and some bars of helium (the pressure broadening enables a better matching between the emission and absorption bandwidths). Xenon can easily be separated from nitrogen and helium via condensation
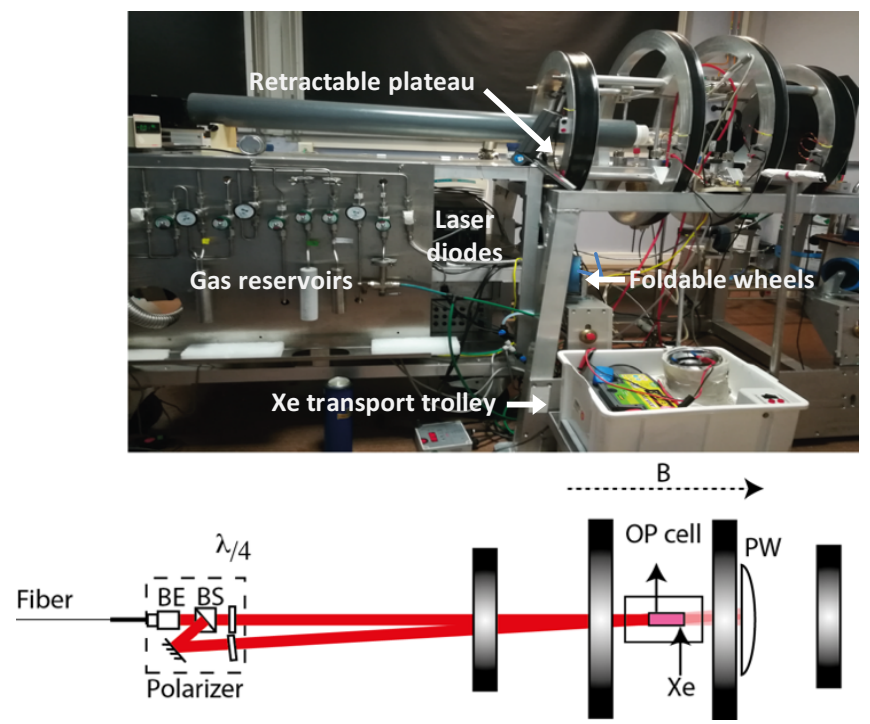

Fig. 2. Production of laser-polarized xenon. Top: Photograph of our SEOP setup; bottom: schematic drawing. BE: beam expander; BS: beam splitter; $\lambda / 4$ : quarter wave plates; PW: powermeter. B: magnetic field ( $\sim 100 \mathrm{G})$ collinear to the light beam.

in liquid nitrogen in the presence of a strong static magnetic field for avoiding fast nuclear relaxation. This step enables hyperpolarized xenon accumulation through multiple optical pumping batches or in flow mode. A concern could appear for the presence of the alkali metal with xenon. At this step, let us mention that inside the cell, at the temperature used during $\mathrm{OP}$ (usually $<100^{\circ} \mathrm{C}$ ), the vapor pressure of, for instance, rubidium, is only 0.2 mTorr. The residual traces of rubidium can thus easily be trapped by passing the mixture through a getter cooled at $-98^{\circ} \mathrm{C}$ by a methanol/liquid nitrogen mixture placed between the OP cell outlet and the storage reservoir. Following these basic principles, Figure 2 displays the schematic drawing of one of our experimental setups. Its additional particularity lies in the removable wheels that enable us to move it easily and place it inside a dedicated van, equipped with a double wall and anoxia detectors. ${ }^{8}$ With this setup, xenon optical pumping can be performed in the near vicinity of the imagers or spectrometers, which reduces the handling risks.

\section{Xenon carriers}

Recent biomolecular magnetic resonance imaging biosensors such as superparamagnetic iron oxide nanoparticles (SPIO) require sufficient quantity, e.g. a concentration of several hundreds of millimolar for mammalian cells, ${ }^{9}$ for efficient diagnosis and treatment and therefore biocompatibility and cytotoxicity exposure are important concerns. Comparatively, 
this new concept of xenon carriers for which fast exchange between free dissolved and complexed xenon provides numerous benefits for in vitro and in vivo applications since only a small quantity of host molecule is required in the sample or tissues, which may drastically decrease the toxicity of the method compared to traditional imaging techniques using magnetic resonance contrast agents. Furthermore, xenon is a noble gas that is de facto inert to most common chemical reactions. As a result, quantities of xenon can be introduced and may lead to repetitive measures, enabling a longitudinal follow-up of biological events, provided that the interaction with the carrier is reversible. Moreover, the powerful benefit of this concept is that a dedicated molecular host specifically alters the resonance frequency of the encapsulated xenon nuclei. For instance, for cryptophanes, due to the large shielding created by the aromatic rings, the ${ }^{129}$ Xe NMR signal corresponding to the caged xenon is shifted from the dissolved xenon signal by more than $130 \mathrm{ppm}$, giving it a unique spectral signature even at moderate magnetic field. ${ }^{10}$

For ${ }^{129} \mathrm{Xe}$ NMR-based biosensing applications, the main challenge is to design xenon carriers with suitable properties toward the hyperpolarized gas. Their first role is to slow down the exchange between two states (free and bound xenon) at the NMR timescale, helped in that by the frequency separation between their signals. The crucial parameter for these host molecules is the in-out xenon exchange rate that must be lower than this frequency separation, but fast enough to enable constant replenishment of the cage in hyperpolarized xenon (see Methods). Also, the large non-equilibrium spin polarization of xenon has to be maintained by using host systems in which the relaxation time is not too fast.

For most in vitro and in vivo applications, these structures must be water-soluble to avoid the formation of selfassemblies such as micelles or vesicles. ${ }^{11}$ Quite a few strategies to render water-soluble these systems, which have in counterpart to contain an hydrophobic core to host xenon, are usually employed: either changing the xenon carrier surface interaction or suitably choosing the recognition antenna.

In this domain, a first strategy was to replace the methyl groups of cryptophane-A by suitable hydrophilic groups such as carboxylate groups. ${ }^{12}$ Another strategy was the metalation of the six arene rings of cryptophane- 111 by [Cp*Ru] moieties leading to cryptophane salts which exhibit a very high water solubility at physiological $\mathrm{pH}$ and the highest xenon affinity ever reported. ${ }^{10}$ More recently, a new cryptophane skeleton has been developed: cryptophane with two ethylenedioxy linkers and the third linker of the propylenedioxy type bearing a unique secondary alcohol. ${ }^{13}$ A second solubilizing or functional group can therefore be selectively introduced, facilitating the synthesis of new molecular platforms with reduced risks of creation of diastereomers.

For the design of ${ }^{129}$ Xe NMR-biosensors, the host systems need to be functionalized for addressing a specific biological target. An important concern can also be the ability of the biosensor to cross the cell membrane.

Considering all these requirements, various host structures have been synthesized during the recent years: cryptophanes, ${ }^{10}$ cucurbiturils, ${ }^{14}$ cucurbituril-based rotaxanes, ${ }^{15}$ pillararenes, ${ }^{16} \mathrm{Fe}_{4} \mathrm{~L}_{6}$ cages $^{14}$ (respectively 1 to 5 in Fig. 3), cyclodextrines, ${ }^{18}$ calixarenes. $^{19}$ For trapping multiple xenon atoms, zeolites ${ }^{20}$, gas vesicles, ${ }^{21}$ nanoemulsions, ${ }^{22}$ bacteriophages ${ }^{23}$ (respectively 6 to 9 in Fig. 3), nanodroplets ${ }^{24}$ or genetically-encoded proteins ${ }^{25}$ have been proposed. These xenon nanocarriers have the particular feature of encapsulating a high number of hyperpolarized xenon atoms, thereby improving the NMR signal. These different structures are the elementary bricks of this powerful generation of ${ }^{129} \mathrm{Xe}$ NMR-based biosensors.

From these xenon carriers, ${ }^{129}$ Xe NMR-based sensors have been conceived following two strategies. Either they will give rise to a signal intensity corresponding to their local density or interaction with their target will lead to a new NMR signal, distinct from the initial one. The concept of density-based biosensors and smart biosensors will now be described.

\section{Density-based biosensors}

To reach biological receptors, the xenon carriers have to be chemically or biologically functionalized with antennas that are able to recognize a specific target. Some studies have noted that the tether between the host molecule and the antenna has a crucial importance. ${ }^{26}$ If the ligand and/or the biological receptor have high molecular weights, a too short or too rigid
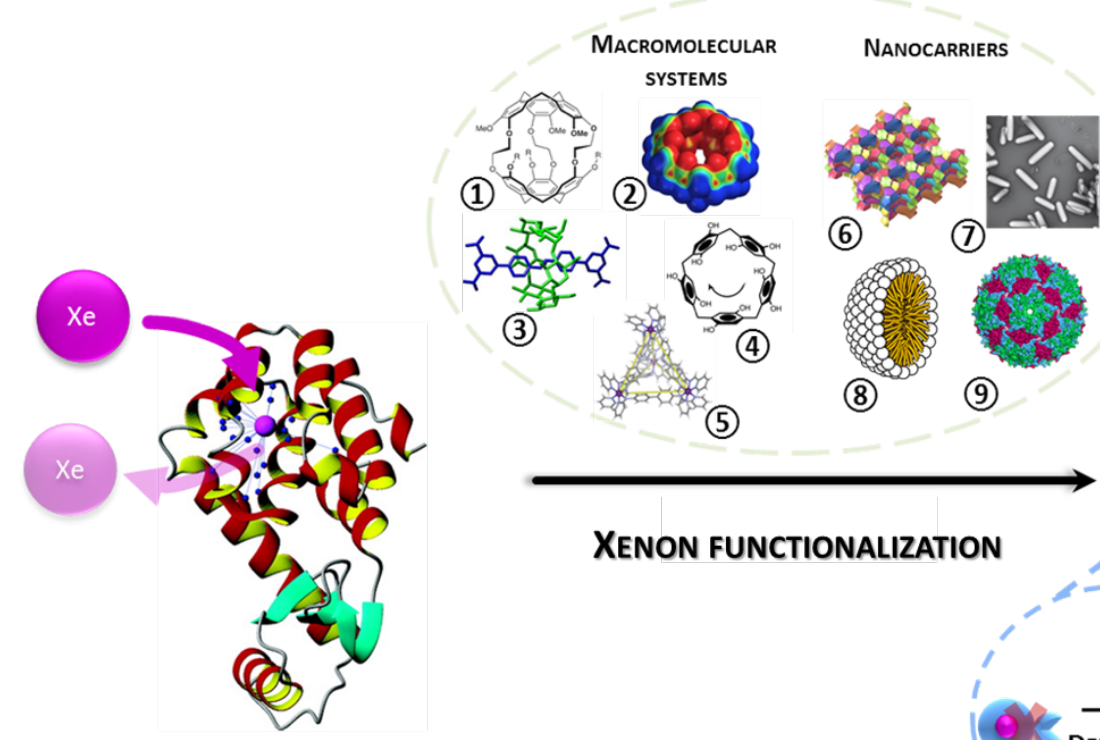

XENON FUNCTIONALIZATION

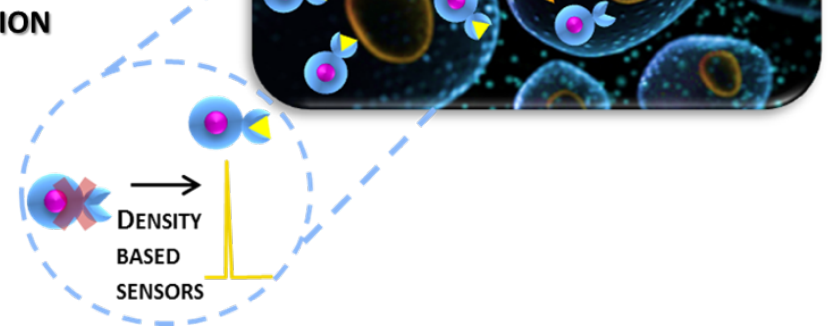

Fig. 3 - Carriers encapsulating hyperpolarized xenon and targeting analytes of interest leading to density-based and smart sensors. 
tether makes that the moiety hosting xenon can have the same correlation time as the macromolecule. This leads to accelerated xenon transverse relaxation, which has the main consequence of broadening the signal of bound xenon and impedes its direct detection.

A first proof-of-principle has been achieved in vitro by the group of Alexander Pines in 2001, detecting avidin using xenon functionalized by a biotinylated supramolecular cage. ${ }^{27}$ Typically, cryptophane-A has been the xenon host of choice and has been subsequently functionalized with peptides for detection of human carbonic anhydrase, ${ }^{28} \alpha_{2 b} \beta_{3}$ integrins, ${ }^{29}$ and major histocompatibility complex (MHC) class II protein. ${ }^{30}$ Meanwhile, due to the difficult synthesis of cryptophanes, the low synthetic yields and most of the time the unavoidable presence of diastereomers, recent studies have turned to cucurbiturils (mainly cucurbit[6]uril). ${ }^{31,32}$ They present a xenon in-out exchange rate faster than cryptophanes, but they are however difficult to chemically substitute and to date no cucurbituril decorated with a ligand has been synthesized. An approach where cucurbituril is used as a molecular relay is rather proposed, where competition between xenon complexation and that of a two-faced guest is used to detect interaction of this guest with the target protein. Such an original strategy unfortunately has an uncertain future for in vivo development, due to the extreme quantity of competitors that can interfere. Furthermore, this structure is only watersoluble at low $\mathrm{pH}^{33,34}$

Since free xenon can cross the plasma membrane without any significant nuclear polarization loss, the targeted receptors can be situated either on the cell surface or inside the cell. Proving that xenon biosensors can be addressed to intracellular markers is a real challenge for in cellulo molecular imaging and can promote the development of intracellular sensors. The first detection via hyperpolarized ${ }^{129}$ Xe NMR of the cell uptake of a biosensor was made with the transferrin system. ${ }^{35}$ Distinct xenon NMR signals corresponding to biosensors in the lipidic part and the aqueous compartment could be evidenced.

Other biosensors were also designed to distinguish between different cell types based on their surface markers, such as specific binding and detection of lymphoma ${ }^{23}$ and cancer cells. $^{36}$

Because in vivo molecular imaging application is the Holy grail, this new hyperpolarized ${ }^{129}$ Xe NMR technology requires a versatile biosensor. Schröder et al. developed a modular construction (see Fig. 4) that allows for quick and easy adaptation of the biosensor to any cell surface target for which there is a specific antibody. ${ }^{37} \mathrm{~A}$ commercially available avidin conjugation kit is used in addition to cryptophane-A monoacid,

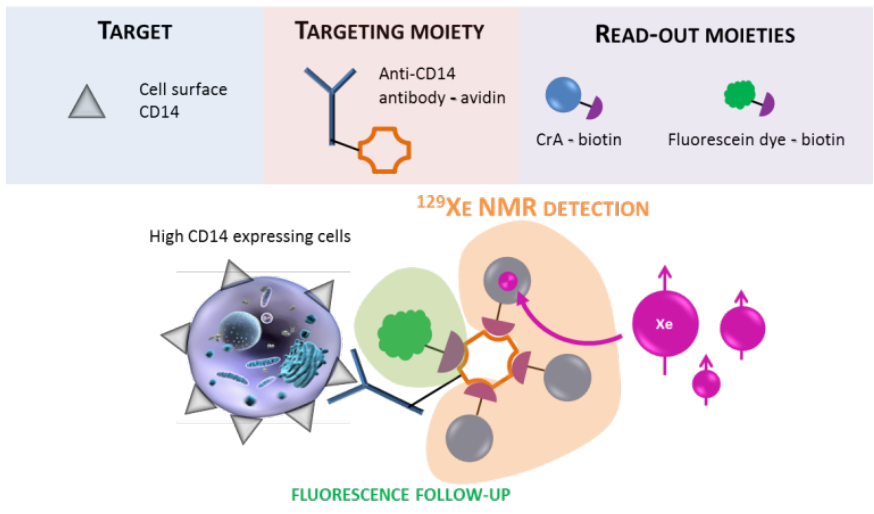

Fig 4 - Modular construction of a sensor based on avidin-conjugated antibody and a set of biotin-conjugated read-out moieties for dual - NMR and fluorescence - functionality. CrA: cryptophane-A. Adapted from ref. 37.

fluorescein and the antibody corresponding to the chosen receptor. It is possible to adapt this system to a variety of biological targets and to detect concentrations of cryptophane-based biosensors as low as $20 \mathrm{nM}$, paving the way to innovative xenon MRI applications.

The spatial resolution of magnetic resonance imaging in its current implementation is limited by the pulsed gradient strength and by xenon transverse relaxation. Due to the ${ }^{129} \mathrm{Xe}$ gyromagnetic ratio ca. 4 times lower than this of proton, reaching a sub-cellular resolution is illusory. Therefore, in order to be able to localize a biosensor inside the cell, bimodal fluorescence - ${ }^{129} \mathrm{Xe}$ NMR biosensors have been conceived. ${ }^{35,37,38,39}$

\section{Smart sensors}

One of the most impressive properties of xenon is its extreme sensitivity towards tiny disruption of its local environment. This sensitivity translates into a very large range of NMR parameter values. In particular the chemical shift of the monoatomic species can span values ranging from $0 \mathrm{ppm}$ (gas phase) to more than $310 \mathrm{ppm}$ (xenon in metalated cryptophanes).

This property can be used to detect small analytes or variation of the physiological environment $(\mathrm{pH}$, temperature), which are markers of major interest for various applications: studying the role of proteins, understanding biological processes, detecting cancer cells, diseases, etc. Today there is a strong demand for highly sensitive analytical methods aiming at the selective and ratiometric detection of these markers.

In analogy to what was developed for MRI contrast agents, ${ }^{40}$ the concept of smart or responsive sensor consists in net modification of the ${ }^{129}$ Xe NMR spectrum when the sensor and its target are in contact, behaving in that as an actuator facilitating detection: instead of (or in addition to) a density- 
based signal, now the signals of the 'free' sensor and of the sensor in interaction with its target are distinct.

Conception of various responsive sensors have allowed specific detection of physical parameter changes $\left(\mathrm{pH},{ }^{41,42}\right.$ temperature $\left.{ }^{43}\right)$, of cations, ${ }^{44-46}$ or of chemical species $\left(\mathrm{H}_{2} \mathrm{O}_{2},{ }^{47}\right.$ rhodamine $6 \mathrm{G},{ }^{48}$ dithiols ${ }^{49}$ ).

The design of molecular systems that enable variation of the bound xenon chemical shift upon complexation of the analyte or variation of the external medium can be achieved by different ways. The use of ionisable groups close to the cavity of cryptophanes leads to $\mathrm{pH}$-sensitive sensors, the caged xenon chemical shift experiencing a large variation in the region of the pKa of these groups. ${ }^{41}$ Also, the grafting of chemical functions that will react with the targeted analyte may lead to significant chemical shift variations for caged xenon (Figure 5). ${ }^{47} \mathrm{~A}$ third category consists in cage-molecules bearing a generic functional group, such as ethylenediaminetetraacetic acid (EDTA), nitrilotriacetic acid (NTA) and tripolyphosphate (TPP), designed to non-specifically chelate metal cations. A hydrophilic cryptophane core bearing a NTA group was shown to be able to chelate $\mathrm{Pb}^{2+}, \mathrm{Zn}^{2+}$ and $\mathrm{Cd}^{2+}$ ions, giving rise to a unique ${ }^{129} \mathrm{Xe}$ spectral signature for each of these ions. ${ }^{44}$ Detection of $\mathrm{Pb}^{2+}$ ions at a concentration of $10 \mathrm{nM}$ was shown possible with a single hyperpolarized xenon batch.

Another design based on cage molecules bearing a nucleotide strand enables detection of the complementary DNA sequence, extending the sensor concept to complex systems, such as DNA hybridization which deals with much weaker bindings $\left(K \approx 10^{6} \mathrm{M}^{-1}\right)$ than the previously studied systems. ${ }^{11}$

A detailed understanding and a reliable modeling of the interactions responsible for such effects on the resonance frequency of caged xenon are therefore of high value. Being able to precisely predict the chemical shift variation of xenon caged in a ${ }^{129}$ Xe NMR-based sensor in response to a specific analyte is a powerful tool for this field. By taking into account the relativistic effects of the interaction with xenon in DFT calculation, a high precision can be achieved in the prediction or simulation of caged xenon chemical shift. In particular, it has been possible to understand the chemical shift evolution of xenon encaged in a cryptophane bearing groups reacting with $\mathrm{H}_{2} \mathrm{O}_{2}$, in clusters separated by $10 \mathrm{ppm}$ according to the progression of the chemical reaction (Fig. 5). ${ }^{47}$

The variations of the caged xenon chemical shift upon activation of the sensor can however be poor, and in case of sample heterogeneity or of broad ${ }^{129}$ Xe signals, be difficult to detect. Therefore the quest for new molecular constructions with which there is modification of another NMR parameter upon target binding is of importance. Some recent works have proposed sensors in which the xenon transverse ${ }^{50}$ or longitudinal ${ }^{51}$ relaxation rates are modified when the construct encounters the target. However, as they give rise to a negative contrast (the signal disappears faster when the target is reached) their applicability for inhomogeneous samples such as cell suspensions or in vivo is not yet demonstrated.
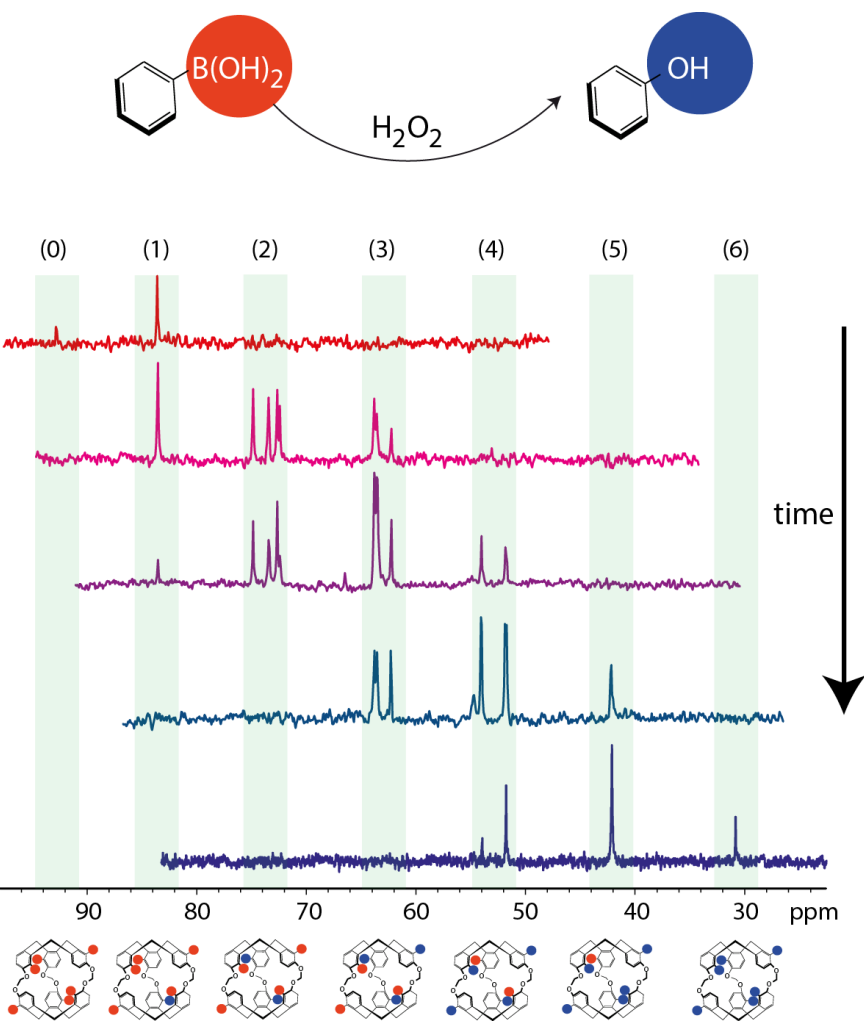

Fig. 5. Time evolution of the ${ }^{129}$ Xe NMR spectrum (high-field region) of xenon caged in a biosensor let in the presence of oxygenated water. Each arylboronate group transformed into phenol group by action of $\mathrm{H}_{2} \mathrm{O}_{2}$ leads to a high field shift variation of the Xe@cage signal by ca. 10 ppm. Such an effect has been understood thanks to DFT calculation including relativistic terms for the interaction. ${ }^{47}$

Another modular platform, based on cucurbituril rotaxanes, ${ }^{52}$ has been developed by the group in Berkeley. ${ }^{15,53}$ For detection of a given enzyme, the terminal part of a peptidic motif known to be cleaved by the enzyme is grafted to a triazole diammonium moiety and a bulky group $\mathrm{R}_{1}$. When left in the presence of cucurbit[6]uril (CB6), it constitutes a rotaxane, which can then be sealed by another bulky group $R_{2}$ (see Figure 6). At this stage, as xenon cannot enter the cavity of CB6, there is no Xe@CB6 signal. This one appears only when $\mathrm{CB} 6$ is released by action of the protease on the peptidic motif, which constitutes a selective activation. This platform can be diversified and developed towards in situ detection of biological events of interest. 


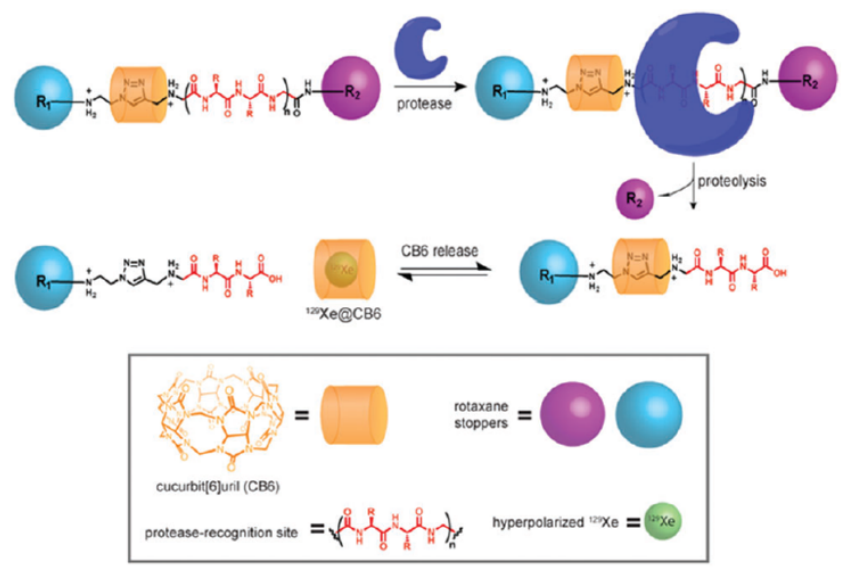

Fig. 6. ${ }^{129}$ Xe NMR-based sensing using a CB6 rotaxane. Reproduced from ref. 53.

\section{Methods}

The high diffusivity of the noble gas combined with its propensity to take a specific chemical shift for every local environment makes that xenon constantly in exchange can be monitored by the various NMR frequencies it takes. This property can be used to further increase the detection sensitivity. Xenon loses its hyperpolarization either progressively via longitudinal relaxation $\left({ }^{129}\right.$ Xe relaxation times from $5 \mathrm{~s}$ to $700 \mathrm{~s}$ have been reported when the noble gas is dissolved in the blood ${ }^{54}$ and in deuterated water, ${ }^{55}$ respectively) or more suddenly when a coherent $\mathrm{rf}$ pulse is applied.

The methods used to detect a small reservoir of hyperpolarized xenon in exchange with a large reservoir can be sorted in two classes: direct and indirect detection. These terms refer to the fact that either the small reservoir signal or variations on the large signal are observed.

In the direct detection methods, fast repetition of spectrallyselective pulses around the small reservoir resonance frequency (and detection after each pulse) uses the chemical exchange between the two environments to gradually increase the signal-to-noise ratio. ${ }^{56,57}$ The repetition rhythm is chosen according to the exchange rate of xenon between the two environments.

The pulse sequence can be written so as to provide a $2 \mathrm{D}$ data matrix, enabling measurement of the apparent relaxation rate in the second dimension and thereby adapting the number of experiments to be summed for obtaining a spectrum with optimized signal-to-noise ratio.

The indirect detection methods - originally proposed by the Pines' group under the acronym HyperCEST - consist of saturation at the small reservoir frequency and detection of the reduction of the large reservoir signal intensity induced by the chemical exchange. Normalized comparison between the signals obtained after off- and on-resonance saturation is sufficient to detect the presence of the small reservoir, but it can also be interesting to recover the whole spectrum through varying the saturation frequency and acquiring data points over a large spectral range (a Z-spectrum). This scheme, available in several sequences, has been used by several authors to detect trace amounts of xenon hosts.

Two sequences have recently been implemented. i) When sensitivity is a concern, the most straightforward sequence is to record depolarization curves - i.e. acquiring spectra for different saturation times - with saturation applied at a frequency close to the bound xenon resonance frequency (on resonance) and far from the free- and bound xenon resonance frequencies (off-resonance), see Fig. 7.

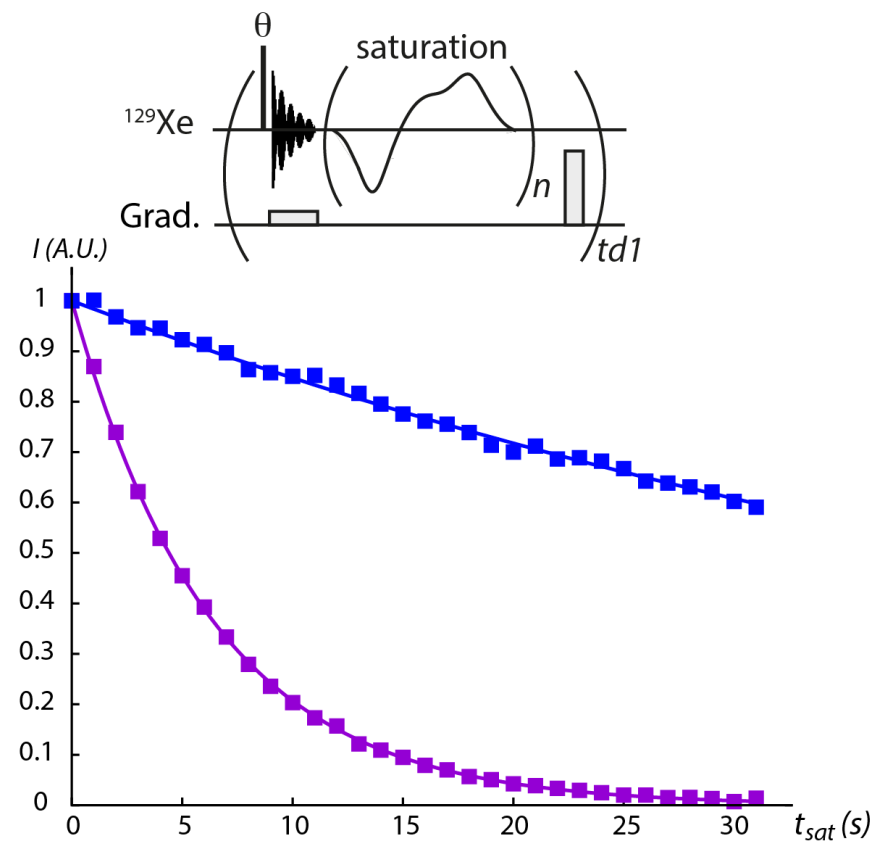

Fig. 7. ${ }^{129}$ Xe HyperCEST depolarization curves. Top: example of pulse sequence (providing a $2 \mathrm{D}$ matrix with each row corresponding to a saturation time $t_{\text {sat }}$ ). A small flip angle $(\theta)$ readout pulse is used (black rectangle), then acquisition is performed in the presence of a field gradient. This gradient is intended to accelerate the transverse relaxation $\left(1 / \mathrm{T}_{2}{ }^{*}\right)$ and therefore enable very short acquisition (on the order of $20 \mathrm{~ms}$, a delay negligible in front of the saturation delay). A short field gradient of random intensity follows saturation in order to remove all magnetization remaining in the transverse plane before the readout pulse (next loop). Bottom: plots of the xenon magnetization as a function of $t_{\text {sat }}$, off-resonance (blue) and on-resonance (purple) (consecutive data points are separated by $0.5 \mathrm{~s}$; saturation: repetition of $100 \mathrm{D}$-SNOB pulses, max. field strength of $12 \mu \mathrm{T}$ ). The sample was a xenon biosensor at $500 \mu \mathrm{M}$ in PBS. 
ii) For its part, the Ultra-Fast Z-spectroscopy enables recording of the Z-spectrum in only two scans. ${ }^{58}$ The principle lies in the application of a field gradient during saturation (enabling saturation of a slice in the sample) and, after the readout pulse, of another field gradient during detection (giving the profile of the sample). Comparison of the profiles obtained with off-resonance and on-resonance saturation directly leads to the Z-spectrum. This is illustrated in Figure 8 on a cryptophane internalized in biological cells.
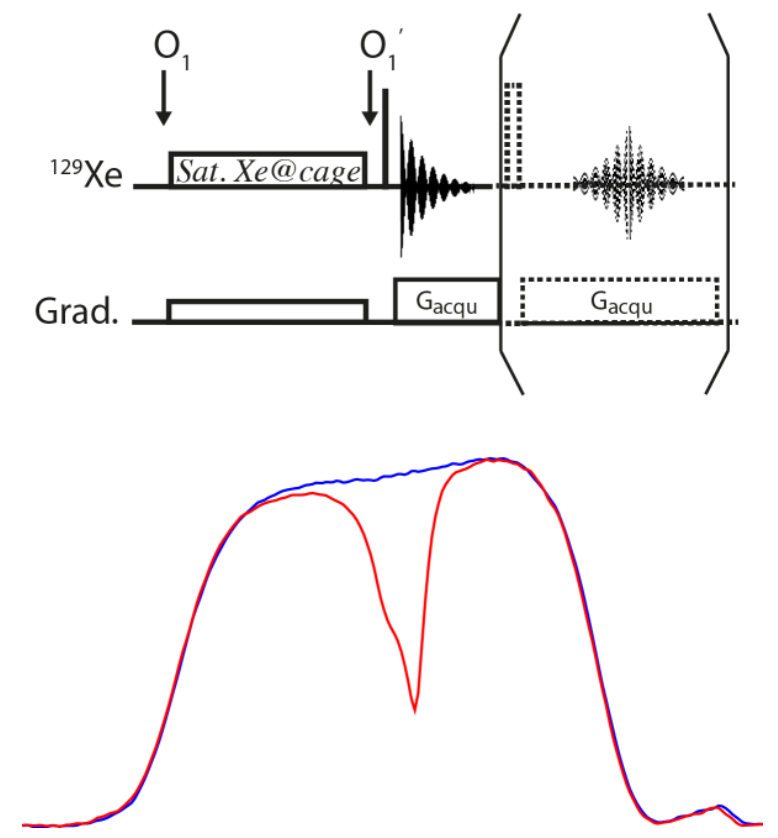

Fig. 8. ${ }^{129}$ Xe Ultra-Fast Z-spectroscopy. Top: used pulse sequence. ${ }^{58}$ The arrow indicate offset changes, from the Xe@host region (O1) to the free xenon signal (O1'). Bottom: a Z-spectrum revealing the presence of a cryptophane into pulmonary A549 cells is extracted in only two scans, by comparison of the profiles obtained with off-resonance saturation (in blue) and on-resonance saturation (in red).

While for the same experiment time and for a large range of exchange rates the HyperCEST-type methods are more sensitive than the direct detection methods (due to the fact that noise is acquired only once), they only provide a dip in the large reservoir signal dependent on the amount of small reservoir but also on the duration of saturation, the relaxation and chemical exchange rates, and the notion of phase is lost. It means that the generation of pulse sequences beyond simple signal observation is less straightforward. For instance, this prevents localized spectroscopy or spectroscopic imaging which are wished to observe the space distribution in the case of several ${ }^{129}$ Xe NMR-based sensors or a smart biosensor delivered in vivo. For a chemical shift imaging (CSI) experiment with the direct detection scheme, the method is simple: addition of two phase gradients after the initial excitation pulse (that can be applied simultaneously with a gradient to obtain a slice selection ${ }^{\ddagger}$ ) provides a 3D experiment with two spatial dimensions and one spectral dimension, in which it is possible to observe the ${ }^{129}$ Xe NMR (sub)spectrum.

Figure 9 displays such an experiment, performed with a single delivery of hyperpolarized xenon before the NMR experiment, where it is possible to localize pixels containing different distribution of water-soluble cryptophanes, at micromolar concentration. Performing such an experiment in the indirect mode would have been much more cumbersome.

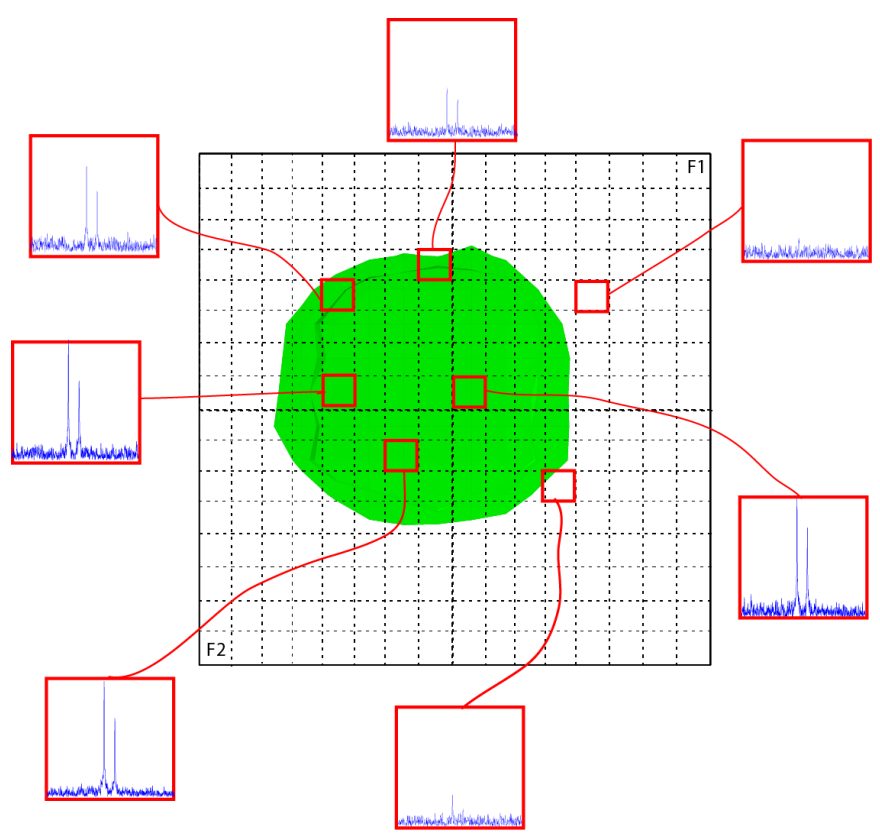

Fig. 9. Hyperpolarized ${ }^{129} \mathrm{Xe} C \mathrm{CS}$ experiment. The sample was an aqueous mixture of two cryptophanes at $100 \mu \mathrm{M}$ in an $8 \mathrm{~mm}$-o.d. NMR tube. The axial imaging is divided in 32x32 points in the spatial dimensions (experiment time $1 \mathrm{~min} 40 \mathrm{~s}$ ).

For the HyperCEST-type methods, a simplistic view would consider that the faster the xenon in-out exchange and the stronger the saturation the more sensitive the detection. However, several other parameters must be taken into account. Denoting $I$ the spin of free xenon and $S$ the spin of encapsulated xenon, of relative proportions $f_{l}$ and $f_{s}=1-f_{l}$, respectively, and the exchange rates $k_{\text {in }}$ and $k_{\text {out }}$ one has: $k_{\text {in }} f_{1}$ $=k_{\text {out }} f_{s}$. An rf irradiation of amplitude $2 \omega_{1}$ applied at a frequency $\omega_{0}$ tilts the magnetization of the two species by an angle $\theta_{l, S}$ from the static field axis in the rotating frame:

$\theta_{l, S}=\arctan \left(\omega_{1} /\left(\omega_{l, S}-\omega_{0}\right)\right)$.

The effective longitudinal relaxation rates along this axis are: $\rho_{l}=\cos ^{2} \theta_{l} R_{1 /}+\sin ^{2} \theta_{l} R_{2 l}$

$\rho_{s}=\cos ^{2} \theta_{s} R_{1 s}+\sin ^{2} \theta_{s} R_{2 s}$

where $R_{1, S}$ and $R_{2 l, S}$ are the pure longitudinal and transverse relaxation rates of spins / or $S .{ }^{59}$ Studying the dynamics of the magnetization can be simplified by considering the secular approximation. The six magnetization components $\left(I_{X}, I_{Y}, I_{Z}, S_{X}\right.$, $S_{Y}, S_{Z}$ ) can be reduced to the two aligned with the effective field axes. They are coupled by the exchange rates $k_{\text {in }}$ and $k_{\text {out }}$ : $\mathrm{d} l_{Z} / \mathrm{d} t=-\left(\rho_{I}+k_{\text {in }}\right) I_{Z}+k_{\text {out }} S_{Z}$ $\mathrm{d} S_{Z} / \mathrm{d} t=-\left(\rho_{S}+k_{\text {out }}\right) S_{Z}+k_{\text {in }} I_{Z}$. 
Here we have considered that the thermal equilibrium magnetization values are negligible with respect to hyperpolarized magnetization.

For an rf saturation applied exactly at the resonance of the small reservoir signal $\left(\omega_{0}=\omega_{s}\right)$ and assuming no spillover effects on the large xenon pool, they simplify to:

$\mathrm{d} l_{z} / \mathrm{d} t=-\left(\mathrm{R}_{1 /}+k_{\text {in }}\right) I_{z}+k_{\text {out }} S_{Z}$

$\mathrm{d} S_{Z} / \mathrm{d} t=-\left(\mathrm{R}_{2 S}+k_{\text {out }}\right) S_{Z}+k_{\text {in }} I_{Z}$.

Considering the evolution of the global xenon magnetization $\mathrm{d}\left(I_{Z}+S_{Z}\right) / \mathrm{d} t=-\mathrm{R}_{1 /} I_{Z}-\mathrm{R}_{2 S} S_{Z}$ it is notable that important parameters are the longitudinal relaxation rate of free xenon and the transverse relaxation rate of bound xenon.

The HyperCEST experiments work in transient mode and take benefit of these couplings for observing differential decay rates of the $I$ spins according to the on- and off-resonance saturation of the $S$ magnetization. Considering that $k_{\text {out }} \gg \mathrm{R}_{2 S}$ $>R_{1 /}$ so that we can neglect the relaxation terms, then the Hyper-CEST efficiency is expressed as: $:^{60,61}$

HyperCEST $\left(\omega_{1}, \omega_{0}, t_{\text {sat }}\right)=1-\exp \left(-k_{\text {out }} f_{S} / f_{l} \alpha\left(\omega_{1}, \omega_{0}\right) t_{\text {sat }}\right)$, where: $\alpha\left(\omega_{1}, \omega_{0}\right)=\omega_{1}{ }^{2} /\left(\omega_{1}{ }^{2}+k_{\text {out }}{ }^{2}+\left(\omega_{S}-\omega_{0}\right)^{2}\right)$ is the saturation efficiency.

Logically, the faster the xenon exchange - the lower the residence time of xenon in its host -, the less efficient the saturation for a given $r$ strength. From this Lorentzian shape with a full width at half maximum equal to $\left(2\left(\omega_{1}{ }^{2}+k_{\text {out }}{ }^{2}\right)\right)^{1 / 2}$, it follows that using an intense $B_{1}$ field has the advantage of maximizing the saturation efficiency, but enlarges the spectral dip which is detected. ${ }^{62}$ In relation with this potential issue, let us mention an experimental problem that can easily occur with high saturation strength with the most rudimentary version of HyperCEST consisting in simply comparing the depolarization response as a function of saturation duration applied on-resonance and off-resonance (at a frequency offset symmetrical with respect to the free xenon signal). Considering the expression of $\rho_{l}$ as a function of $\omega_{1}$, the large difference between longitudinal and transverse xenon relaxation and the fact that temperature increase and BlochSiegert effects can slightly shift the free xenon signal, this can easily induce a difference between the on- and off-resonance cases even in the absence of xenon host.

Actually for most of the xenon hosts and particularly cryptophanes, it has been observed that the line width of the caged xenon signal is influenced by the xenon concentration, $^{63,64}$ which is the signature of a degenerate exchange $^{65}$ (or kick-out) mechanism, where a caged xenon atom is expelled by another xenon atom. The shortening of the caged xenon lifetime depends on the ratio of the noble gas concentration over the concentration of the host. Therefore, the Hyper-CEST efficiency will strongly depend on this ratio, and the saturation strength and duration will need to be adjusted according to the previous expressions. ${ }^{62}$

\section{Future directions}

Efforts are now focused on extending this recent concept of ${ }^{129}$ Xe NMR-based biosensing to in vivo applications since xenon can cross the pulmonary alveolar-capillary barrier and other multilayers such as the blood-brain barrier without losing too much hyperpolarization. But the passage to real in vivo experiments requires some adaptations. Both the protocols to deliver hyperpolarized xenon and the NMR/MRI methods depend on the targeted organ. It is expected that the local density of free hyperpolarized xenon will be lower than for in vitro cases, and that the longitudinal relaxation rate of xenon outside the host may be short (shorter than in the host + ), resulting in a poorer signal to noise ratio. Moreover, in vivo, many analytes, ions etc. can compete with xenon for the interaction with the host molecule. Finally, due to constraints linked to the specific absorption rate (SAR), the radiofrequency deposition must be limited. Recent studies demonstrated that, compared to a continuous wave, a band-selective saturation sequence based on multiple pulse inversion elements can yield saturation bandwidth tunable over a wide range, while inducing less RF power in the sample and therefore preventing RF tissue heating during imaging. ${ }^{66}$

Thus, according to the remarks done in the previous Sections, the quest for a host molecule for which xenon exhibits a strong affinity - and still a high in-out exchange - is made important again. In this sense, so far cryptophanes seem to be the best candidates, as they could provide the higher contrast at low saturation strength. ${ }^{32, \$}$

Additional issues such as delivery of hyperpolarized xenon and biosensors to the receptors of interest also need to be noted. Hyperpolarized xenon can be delivered into the bloodstream by injection in physiological solutions or into lung and brain through inhalation. ${ }^{67}$

Another main challenge in in vivo molecular imaging is that modalities with the highest sensitivity have relatively poor spatial resolution, while those with high resolution have relatively poor sensitivity. The idea of combining multiple modalities has grown in the past few years and researchers have come to realize that complementary abilities of different imaging techniques could be exploited by using them in tandem. The new generation of sensors trends towards host systems, combining complementary molecular imaging techniques, able to target a specific receptor and furthermore to be activated by its presence. Tsien and his group have proposed a moiety which can specifically bind with high affinity a Cys-tag protein motif (Cys-Cys-X-X-Cys-Cys, where $X$ can be any residue) and that turn fluorescent upon complexation. ${ }^{68}$ Grafting this small responsive ligand on a hexacarboxylated cryptophane-A therefore allows a bimodal detection of tagged protein, giving rise to activated signals in both fluorescence and NMR. ${ }^{69}$ This doubly smart sensor gave 
interesting results for detection of model peptides tagged with the Cys4 motif. Detection of recombinant proteins in cellulo with this method is underway in our laboratory. Another doubly smart construction based on the same principle has been developed by Zhou and co-workers for detection of biothiols in biological cells. ${ }^{70}$

Finally, biosensors leading to multiplexed detection should be investigated in the future in order to simultaneously visualize multiple markers and therefore be a powerful tool for disease diagnosis.

Hyperpolarized xenon has amazing properties and its combination with host molecules makes it a unique system for longitudinal follow-up of biological events. The principle is rather different from strategies based on delivery of species hyperpolarized by DNP or by para-hydrogen, which are limited by their longitudinal relaxation time. Here the approach consists in a first step to deliver the xenon biosensor, which will hopefully reach its target. Then, in a second step fully decorrelated from the first one, hyperpolarized xenon can be introduced and imaging can be performed. This last operation can be repeated many times. As for the final objective which is to follow pathology in vivo through this approach, ${ }^{129}$ Xe NMRbased sensors offer a huge potential and applications are finally at hand.

\section{Conflicts of interest}

There are no conflicts of interest to declare.

\section{Acknowledgements}

The authors are indebted to Pr. Hervé Desvaux for a careful reading of this manuscript. This work is supported by a public grant overseen by the French National research Agency (ANR) as part of the "Investissement d'Avenir" program, through the "IDI 2017" project funded by the IDEX Paris-Saclay, ANR-11IDEX-0003-02.

\section{Notes and references}

\# There is a limitation for the slice gradient strength, arising from the fact that the large reservoir signal should not be excited. But it is not stringent in most of the cases, given the large spectral separation between the free and caged xenon signals.

$\S$ Another argument for the use of cryptophanes as ${ }^{129}$ Xe NMRbased biosensors in vivo related to the SAR lies in the large frequency separation between the free dissolved xenon signal ( 200 ppm) and the caged xenon signal (around $60 \mathrm{ppm})$, which enables the work at low magnetic field, thereby lowering the SAR.

† The longitudinal relaxation time of hyperpolarized xenon when caged in a cryptophane is about $15 \mathrm{~s}$, while it is shorter in blood (ca. $5 \mathrm{~s}$ ).

1 P. Nikolaou, B. M. Goodson and E. Y. Chekmenev, Chem. - Eur. J., 2015, 21, 3156-3166.
2 C. Boutin, H. Desvaux, M. Carrière, F. Leteurtre, N. Jamin, Y. Boulard and P. Berthault, NMR Biomed., 2011, 24, 1264-1269.

3 T. Chupp and S. Swanson, Adv. At. Mol. Opt. Phys., 2001, 45, 4198.

4 C. Landon, P. Berthault, F. Vovelle and H. Desvaux, Protein Sci., 2001, 10, 762-770.

5 L. Dubois, P. Da Silva, C. Landon, J. G. Huber, M. Ponchet, F. Vovelle, P. Berthault and H. Desvaux, J. Am. Chem. Soc., 2004, 126, 15738-15746.

6 O. Delhaye, E. Robin, J. E. Bazin, J. Ripart, G. Lebuffe and B. Vallet, Ann. Fr. Anesth. Reanim., 2010, 29, 635-641.

7 A. Capozzi, C. Roussel, A. Comment and J.-N. Hyacinthe, J. Phys. Chem. C, 2015, 119, 5020-5025.

8 C. Chauvin, L. Liagre, C. Boutin, E. Mari, E. Léonce, G. Carret, B. Coltrinari and P. Berthault, Rev. Sci. Instrum., 2016, 87, 016105.

9 M. Mahmoudi, H. Hofmann, B. Rothen-Rutishauser and A. PetriFink, Chem. Rev., 2012, 112, 2323-2338.

10 R. M. Fairchild, A. I. Joseph, K. T. Holman, H. A. Fogarty, T. Brotin, J. P. Dutasta, C. Boutin, G. Huber and P. Berthault, J. Am. Chem. Soc., 2010, 132, 15505-15507.

11 V. Roy, T. Brotin, J.-P. Dutasta, M.-H. Charles, T. Delair, F. Mallet, G. Huber, H. Desvaux, Y. Boulard and P. Berthault, ChemPhysChem, 2007, 8, 2082-2085.

12 G. Huber, T. Brotin, L. Dubois, H. Desvaux, J. P. Dutasta and P. Berthault, J. Am. Chem. Soc., 2006, 128, 6239-6246.

13 L.-L. Chapellet, J. R. Cochrane, E. Mari, C. Boutin, P. Berthault and T. Brotin, J. Org. Chem., 2015, 80, 6143-6151.

14 F. T. Hane, T. Li, P. Smylie, R. M. Pellizzari, J. A. Plata, B. DeBoef and M. S. Albert, Sci. Rep., 2017, 7, 41027.

15 J. A. Finbloom, C. C. Slack, C. J. Bruns, K. Jeong, D. E. Wemmer, A. Pines and M. B. Francis, Chem. Commun., 2016, 52, 31193122.

16 T. Adiri, D. Marciano and Y. Cohen, Chem. Commun., 2013, 49, 7082-7084.

17 J. Roukala, J. Zhu, C. Giri, K. Rissanen, P. Lantto and V.-V. Telkki, J. Am. Chem. Soc., 2015, 137, 2464-2467.

18 Y.-Q. Song, B. M. Goodson, R. E. Taylor, D. D. Laws, G. Navon and A. Pines, Angew. Chem. Int. Ed. Engl., 1997, 36, 2368-2370.

19 J. Fukutomi, Y. Adachi, A. Kaneko, A. Kimura and H. Fujiwara, J. Incl. Phenom. Macrocycl. Chem., 2007, 58, 115-122.

20 F. Lerouge, O. Melnyk, J.-O. Durand, L. Raehm, P. Berthault, G. Huber, H. Desvaux, A. Constantinesco, P. Choquet, J. Detour and M. Smaïhi, J. Mater. Chem., 2008, 19, 379-386.

21 M. G. Shapiro, R. M. Ramirez, L. J. Sperling, G. Sun, J. Sun, A. Pines, D. V. Schaffer and V. S. Bajaj, Nat. Chem., 2014, 6, 629634.

22 T. K. Stevens, R. M. Ramirez and A. Pines, J. Am. Chem. Soc., 2013, 135, 9576-9579.

23 K. Jeong, C. Netirojjanakul, H. K. Munch, J. Sun, J. A. Finbloom, D. E. Wemmer, A. Pines and M. B. Francis, Bioconjug. Chem., 2016, 27, 1796-1801.

24 S. Klippel, C. Freund and L. Schröder, Nano Lett., 2014, 14, 5721-5726.

25 Y. Wang, B. W. Roose, E. J. Palovcak, V. Carnevale and I. J. Dmochowski, Angew. Chem. Int. Ed. Engl., 2016, 128, 91309133.

26 T. J. Lowery, S. Garcia, L. Chavez, E. J. Ruiz, T. Wu, T. Brotin, J.-P. Dutasta, D. S. King, P. G. Schultz, A. Pines and D. E. Wemmer, ChemBioChem, 2006, 7, 65-73.

27 M. M. Spence, S. M. Rubin, I. E. Dimitrov, E. J. Ruiz, D. E. Wemmer, A. Pines, S. Q. Yao, F. Tian and P. G. Schultz, Proc. Natl. Acad. Sci. U. S. A., 2001, 98, 10654-10657. 
28 J. M. Chambers, P. A. Hill, J. A. Aaron, Z. Han, D. W. Christianson, N. N. Kuzma and I. J. Dmochowski, J. Am. Chem. Soc., 2009, 131, 563-569.

29 G. K. Seward, Y. Bai, N. S. Khan and I. J. Dmochowski, Chem. Sci., 2011, 2, 1103-1110.

30 A. Schlundt, W. Kilian, M. Beyermann, J. Sticht, S. Günther, S. Höpner, K. Falk, O. Roetzschke, L. Mitschang and C. Freund, Angew. Chem. Int. Ed., 2009, 48, 4142-4145.

31 Y. Wang and I. J. Dmochowski, Chem. Commun., 2015, 51, 89828985.

32 M. Kunth, C. Witte, A. Hennig and L. Schroeder, Chem. Sci., $2015,6,6069-6075$.

33 Y. Wang, B. W. Roose, J. P. Philbin, J. L. Doman and I. J. Dmochowski, Angew. Chem. Int. Ed., 2016, 55, 1733-1736.

34 M. Schnurr, J. Sloniec-Myszk, J. Döpfert, L. Schröder and A. Hennig, Angew. Chem. Int. Ed., 2015, 54, 13444-13447.

35 C. Boutin, A. Stopin, F. Lenda, T. Brotin, J.-P. Dutasta, N. Jamin, A. Sanson, Y. Boulard, F. Leteurtre, G. Huber, A. BogaertBuchmann, N. Tassali, H. Desvaux, M. Carrière and P. Berthault, Bioorg. Med. Chem., 2011, 19, 4135-4143.

36 K. K. Palaniappan, R. M. Ramirez, V. S. Bajaj, D. E. Wemmer, A. Pines and M. B. Francis, Angew. Chem. Int. Ed., 2013, 52, 48494853.

37 H. M. Rose, C. Witte, F. Rossella, S. Klippel, C. Freund and L. Schröder, Proc. Natl. Acad. Sci., 2014, 111, 11697-11702.

38 G. K. Seward, Q. Wei and I. J. Dmochowski, Conjug. Chem., 2008, 19, 2129-2135.

39 C. Witte, V. Martos, H. M. Rose, S. Reinke, S. Klippel, L. Schröder and C. P. R. Hackenberger, Angew. Chem. Int. Ed., 2015, 54, 2806-2810.

40 C. S. Bonnet and É. Tóth, Chimia, 2016, 70, 102-108.

41 P. Berthault, H. Desvaux, T. Wendlinger, M. Gyejacquot, A. Stopin, T. Brotin, J.-P. Dutasta and Y. Boulard, Chem. - Eur. J., 2010, 16, 12941-12946.

42 B. A. Riggle, Y. Wang and I. J. Dmochowski, J. Am. Chem. Soc., 2015, 137, 5542-5548.

43 F. Schilling, L. Schröder, K. K. Palaniappan, S. Zapf, D. E. Wemmer and A. Pines, ChemPhysChem, 2010, 11, 3529-3533.

44 N. Tassali, N. Kotera, C. Boutin, E. Léonce, Y. Boulard, B. Rousseau, E. Dubost, F. Taran, T. Brotin, J.-P. Dutasta and P. Berthault, Anal. Chem., 2014, 86, 1783-1788.

45 Q. Guo, Q. Zeng, W. Jiang, X. Zhang, Q. Luo, X. Zhang, L.-S. Bouchard, M. Liu and X. Zhou, Chem. - Eur. J., 2016, 22, 39673970.

46 K. Jeong, C. C. Slack, C. C. Vassiliou, P. Dao, M. D. Gomes, D. J. Kennedy, A. E. Truxal, L. J. Sperling, M. B. Francis, D. E. Wemmer and A. Pines, ChemPhysChem, 2015, 16, 3573-3577.

47 E. Dubost, J.-P. Dognon, B. Rousseau, G. Milanole, C. Dugave, Y. Boulard, E. Léonce, C. Boutin and P. Berthault, Angew. Chem. Int. Ed., 2014, 53, 9837-9840.

48 P. D. Garimella, T. Meldrum, L. S. Witus, M. Smith, V. S. Bajaj, D. E. Wemmer, M. B. Francis and A. Pines, J. Am. Chem. Soc., 2014, 136, 164-168.

49 S. Yang, Y. Yuan, W. Jiang, L. Ren, H. Deng, L. S. Bouchard, X. Zhou and M. Liu, Chem. - Eur. J., 2017, n/a-n/a.
50 M. D. Gomes, P. Dao, K. Jeong, C. C. Slack, C. C. Vassiliou, J. A. Finbloom, M. B. Francis, D. E. Wemmer and A. Pines, J. Am. Chem. Soc., 2016, 138, 9747-9750.

51 F. Zamberlan, C. Lesbats, N. J. Rogers, J. L. Krupa, G. E. Pavlovskaya, N. R. Thomas, H. M. Faas and T. Meersmann, ChemPhysChem, 2015, 16, 2294-2298.

52 C. Ke, R. A. Smaldone, T. Kikuchi, H. Li, A. P. Davis and J. F. Stoddart, Angew. Chem. Int. Ed., 2013, 52, 381-387.

53 C. C. Slack, J. A. Finbloom, K. Jeong, C. J. Bruns, D. E. Wemmer, A. Pines and M. B. Francis, Chem. Commun., 2017, 53, 10761079.

54 A. Bifone, Y.-Q. Song, R. Seydoux, R. E. Taylor, B. M. Goodson, T. Pietrass, T. F. Budinger, G. Navon and A. Pines, Proc. Natl. Acad. Sci. USA, 1996, 93, 12932-12936.

55 I. E. Dimitrov, R. Reddy and J. S. Leigh, J. Magn. Reson., 2000, 145, 302-306.

56 S. Garcia, L. Chavez, T. J. Lowery, S.-I. Han, D. E. Wemmer and A. Pines, J. Magn. Reson., 2007, 184, 72-77.

57 N. Kotera, N. Tassali, E. Léonce, C. Boutin, P. Berthault, T. Brotin, J.-P. Dutasta, L. Delacour, T. Traoré, D.-A. Buisson, F. Taran, S. Coudert and B. Rousseau, Angew. Chem. Int. Ed., 2012, 51, 4100-4103.

58 C. Boutin, E. Léonce, T. Brotin, A. Jerschow and P. Berthault, J. Phys. Chem. Lett., 2013, 4, 4172-4176.

59 H. Desvaux and P. Berthault, Prog. Nucl. Magn. Reson. Spectrosc., 1999, 35, 295-340.

60 E. Vinogradov, A. D. Sherry and R. E. Lenkinski, J. Magn. Reson., 2013, 229, 155-172.

61 M. Kunth, C. Witte and L. Schröder, J. Chem. Phys., 2014, 141, 194202.

62 M. Kunth, C. Witte and L. Schröder, NMR Biomed., 2015, 28, 601-606.

63 K. Bartik, M. Luhmer, J.-P. Dutasta, A. Collet and J. Reisse, J. Am. Chem. Soc., 1998, 120, 784-791.

64 G. Huber, L. Beguin, H. Desvaux, T. Brotin, H. A. Fogarty, J.-P. Dutasta and P. Berthault, J. Phys. Chem. A, 2008, 112, 1136311372.

65 S. Korchak, W. Kilian and L. Mitschang, Chem. Commun., 2015, 51, 1721-1724.

66 T. Meldrum, V. S. Bajaj, D. E. Wemmer and A. Pines, J. Magn. Reson., 2011, 213, 14-21.

67 M. L. Mazzanti, R. P. Walvick, X. Zhou, Y. Sun, N. Shah, J. Mansour, J. Gereige and M. S. Albert, PLOS ONE, 2011, 6, e21607.

68 B. A. Griffin, S. R. Adams and R. Y. Tsien, Science, 1998, 281, 269-272.

69 N. Kotera, E. Dubost, G. Milanole, E. Doris, E. Gravel, N. Arhel, T. Brotin, J.-P. Dutasta, J. Cochrane, E. Mari, C. Boutin, E. Léonce, P. Berthault and B. Rousseau, Chem. Commun., 2015, 51, 11482-11484.

70 J. Zhang, X. Bao, J. Zhou, F. Peng, H. Ren, X. Dong and W. Zhao, Biosens. Bioelectron., 2016, 85, 164-170. 